TÉOROS $\begin{aligned} & \text { Téoros } \\ & \text { Revue de recherche en tourisme }\end{aligned}$

$34,1-2 \mid 2015$

Le tourisme hors des sentiers battus

\title{
Au détour des barres et des tours
}

Les quartiers nord de Marseille, de zone à zone touristique?

\section{Yannick HASCOËT et Isabelle LEFORT}

\section{revues.org}

Édition électronique

URL : http://teoros.revues.org/2768

ISSN : 1923-2705

\section{Éditeur \\ Presses de l'Université du Québec}

Ce document vous est offert par SCD Université Lumière Lyon 2

\section{UNR-RA $=$}

Référence électronique

Yannick HASCOËT et Isabelle LEFORT, «Au détour des barres et des tours », Téoros [En ligne], 34,

1-2 | 2015, mis en ligne le 15 mars 2015, consulté le 02 octobre 2017. URL : http://

teoros.revues.org/2768

Ce document a été généré automatiquement le 2 octobre 2017.

\section{c) (†) $९$}

La revue Téoros est mise à disposition selon les termes de la Licence Creative Commons Attribution Pas d'Utilisation Commerciale - Pas de Modification 4.0 International. 


\title{
Au détour des barres et des tours
}

Les quartiers nord de Marseille, de zone à zone touristique?

\author{
Yannick HASCOËT et Isabelle LEFORT
}

1 Les mutations touristiques contemporaines font valoir une mobilisation de nouveaux espaces (les quartiers populaires) et de nouvelles pratiques (participatives). À cet égard, le quartier de Belleville à Paris est un laboratoire exemplaire (Chapuis, 2009). Ces nouvelles modalités touristiques traduisent une évolution culturelle conjointe à certaines dynamiques propres de l'offre touristique. Si ces espaces d'habitat populaire ont longtemps fait partie du négatif urbain mis en tourisme (qu'aller faire dans ces quartiers ?), ils sont aujourd'hui l'objet de «fronts» touristiques, qui en manifestent l'annexion spatiale et sociale aux offres urbaines traditionnelles, voire patrimonialement labellisées. Cette ligne de front se déplace, à présent, des quartiers populaires péricentraux aux quartiers nettement périphériques de banlieue. Certes, ces quartiers de grands ensembles, de barres et de tours, emblématiques de l'urbanisation fonctionnaliste des décennies 1950, 1960 et 1970, souffrent depuis longtemps d'une situation critique (pauvreté économique et sociale, relégation territoriale) et de représentations ellesmêmes péjorées et péjoratives. Or, dans certains de ces mêmes espaces se développent aujourd'hui des pratiques de découverte, de visite et de valorisation s'inscrivant dans un processus de connaissance et donc de reconnaissance de leurs identités architecturale, sociale et culturelle (Vayssière, 2002 ; Pouvreau, 2011 ; Veschambre, 2013).

2 Cette trajectoire fait émerger et mobilise de nouvelles pratiques, tantôt touristiques, tantôt artistiques, qui se combinent parfois : nous présenterons ici ces pratiques « récréa (r)tistes » d'un nouveau genre. Toutefois, mise en scène et parcours ont de quoi surprendre, tant le décalage est effectivement grand entre un imaginaire touristique dominant - sites plaisants et accueillants (Amirou, 1995) - et ces espaces, objets d'une imagerie urbano-pathogène (Avenel, 2004). Dans l'imaginaire collectif, ces quartiers sont, au mieux, sans intérêt, au pire, synonymes de menaces. Tout concourt à associer ces territoires à une banlieue hors des sentiers de l'intéressant. Nous explorons ici les acteurs concernés (visiteurs, "guides ", hébergeurs, artistes) et les mises en œuvre dans les quartiers nord de Marseille, qui élaborent et proposent ces offres d'un nouveau genre 
pour rendre compte et analyser le statut, la fonction et les enjeux de ces pratiques. Ces dernières, en procédant à des mélanges de genres (lieux touristiques estampillés par opposition à marges de découvertes, hébergement en cité opposé à bastides provençales, tourisme ou projet politique) ne manquent évidemment pas d'interroger les catégories d'approche et d'analyse longtemps usuelles de l'objet « tourisme ».

\section{Hôtel du Nord : centre de gravité des pratiques au nord de Marseille}

3 La carte touristique de la ville de Marseille (voir illustration 1) montre combien l'offre touristique marseillaise des acteurs territoriaux promeut essentiellement les lieux, les sites et les déambulations dans les centres de la cité. Le pluriel ici s'impose dans la mesure où à la ville et ses hauts lieux traditionnels (Roncayolo, 1990) (Vieux-Port, Notre-Dame de la Garde, cité radieuse de Le Corbusier, palais Longchamp) se sont récemment ajoutés les quartiers réhabilités et gentrifiés (le Panier, les docks de La Joliette). Ce faisant, la carte de la ville distribuée par l'office de tourisme ignore un pan entier de son territoire et des ressources qu'il abrite. Sauf un encart pour l'Estaque (non reproduit ici), les quartiers nord de Marseille n'ont pas droit à la représentation officielle et promotionnelle, soit une exclusion de près de 8000 hectares et d'environ 250000 habitants.

Or, le collectif habitant « Hôtel du Nord » propose :

Au nord de la capitale phocéenne, là où les barres HLM côtoient les vieilles bastides, se cachent des trésors d'histoire et de culture que leurs habitants redécouvrent et nous invitent à redécouvrir. Depuis plusieurs années, ils fouillent, cherchent, et interrogent un patrimoine foisonnant et souvent méconnu ${ }^{1}$.

Si les quartiers nord de Marseille ne sauraient se réduire aux seules cités d'habitat social, le catalogue s'est enrichi en 2013 de prestations spécifiques, sous le titre «Terroir des Cités » :

«Terroirs des Cités » car la terre des grands ensembles a aussi son histoire, souvent passionnante, sédimentée dans la diversité des parcours humains et des actes urbains qui composent la ville. Des habitants qui vivent là vous invitent à écouter et à partager des espaces, des paysages, des trajectoires de vie et de langues, des histoires d'exil, en un mot leur patrimoine ${ }^{2}$. 
Illustration 1 : Carte touristique de la ville de Marseille

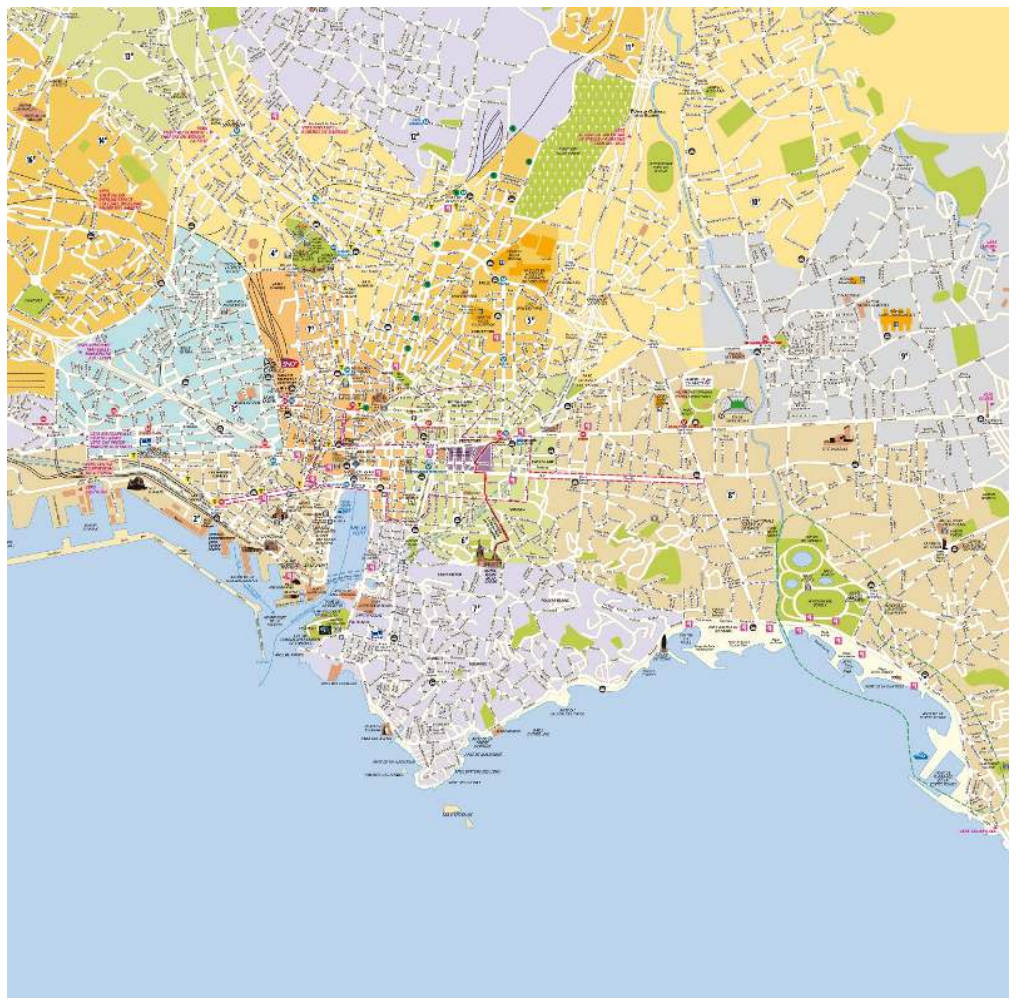

Carte produite et distribuée par les opérateurs territoriaux. Les quartiers nord de la ville sont largement occultés par la représentation officielle.

Source : Office de tourisme et des congrès de Marseille

ILLUSTRATION 2 : Un groupe en visite à la cité de La Visitation dans le cadre de l'Offre « Terroir des cités » de l'Hôtel du Nord

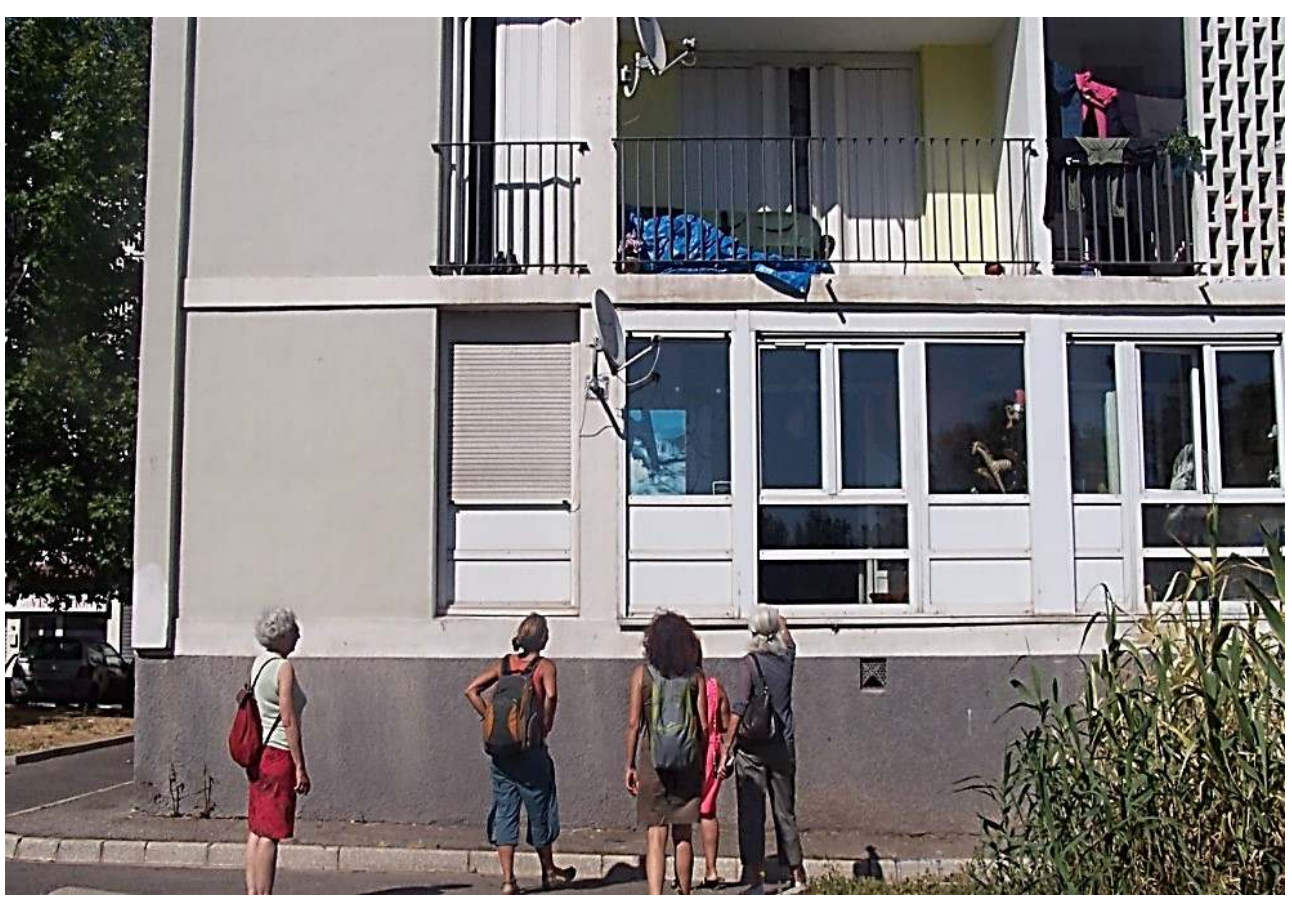

Photographie : Yannick Hascoët, juin 2013 
6 de promotion (dans le cadre des Journées européennes du patrimoine ${ }^{3}$ ) des ressources du nord de la cité phocéenne. Initié au milieu des années 1990 par une historienne alors nommée conservatrice du patrimoine sur ce territoire du Grand Projet de Ville (GPU) ${ }^{4}$, ce projet se retrouve conforté par l'adhésion (2009) de la mairie socialiste du $8^{\mathrm{e}}$ secteur $\left(15^{\mathrm{e}}\right.$ et $16^{\mathrm{e}}$ arrondissements de Marseille) à la Convention européenne de Faro sur la valeur du patrimoine culturel pour la société. Rappelons que les trois principes qui en régissent l'octroi sont le droit au patrimoine, la gestion durable et la gouvernance démocratique. La signature de cette convention amplifie dès lors le processus de patrimonialisation des quartiers nord de la ville. Fort de la perspective de Marseille capitale de la culture $2013^{5}$, les énergies se focalisent sur l'élaboration de la Société coopérative de production (SCOP) Hôtel du Nord: une offre de chambres d'hôtes et de balades urbaines se fait jour. Aujourd'hui, près de 50 sociétaires, dont plus de la moitié sont des habitants, animent un réseau d'environ 50 chambres d'hôtes et autant de balades urbaines ${ }^{6}$.

offre constitue un matériau de choix pour saisir trois systèmes d'hybridation qui agissent et rétroagissent à l'intérieur d'un système où acteurs (offre et demande) et objectifs (faire connaître pour se faire reconnaître) se combinent de façon innovante, « pionnière » selon Michèle Jolé (2012). Cette dernière replace néanmoins fort justement l'offre marseillaise dans un contexte touristique international et national, faisant écho aux expériences participatives des Greeters new-yorkais, au programme «Douce Banlieue » du Comité départemental du tourisme (CDT) de la Seine-Saint-Denis ou de l'association «Jeunes guides en banlieue» pilotée par le Conseil d'architecture, d'urbanisme et de l'environnement (CAUE) du Val-de-Marne. Ajoutons l'« organisation cousine ", selon Hôtel du Nord, qui développe un réseau de chambres d'hôtes en SeineSaint-Denis: Accueil Banlieue. Sur ce territoire inaccoutumé, la double polarité de catégories historiquement antagonistes se re-combine - amateur/professionnel, visiteur/ visité, centre/périphérie - et engendre une offre et des pratiques de visites dont l'hybridation concerne les modalités, les lieux et les registres d'activités.

\section{De vieux couples défaits}

8 Seules deux balades urbaines estampillées «Terroir des Cités " sont pour l'heure régulièrement proposées, par deux guides, dans les cités du Castellas et de La Visitation (illustration 3). Sur le même modèle, une autre balade en cours d'élaboration parcourra la cité des Aygalades, tandis que d'autres abordent les grands ensembles, ainsi celle intitulée « Des bastides à l'habitat social » (sans que les guides soient des habitants des cités). De fait, la coopérative peine à recruter des habitants de cités désireux de jouer les guides.

Sans faire ici une analyse sociologique approfondie des principaux acteurs de la coopérative, leur caractérisation dessine des profils dont les éléments suivants se dégagent. Appartenant bien souvent aux milieux artistique et intellectuel, ils affichent des valeurs éthiques (anti-racisme, vivre-ensemble, écologie, etc.) et sont richement dotés en capitaux culturels (Bourdieu, 1979). Ils apprécient l'ambiance populaire et multiculturelle des quartiers nord, dont ils sont des habitants anciennement installés pour la plupart (dans les noyaux ouvriers villageois anciens ou dans des bastides provençales bourgeoises). Au hasard d'une rencontre, une habitante de la cité du Plan 
d'Aou, toutefois proche d'Hôtel du Nord, nous confiera : « Nous, les gens d'Hôtel du Nord on les appelle les bac + 5 » (la cinquantaine, cuisinière, d'origine algérienne, janvier 2014).

\section{Illustration 3 : La géographie « récéa(r)tiste » des quartiers nord de Marseille}

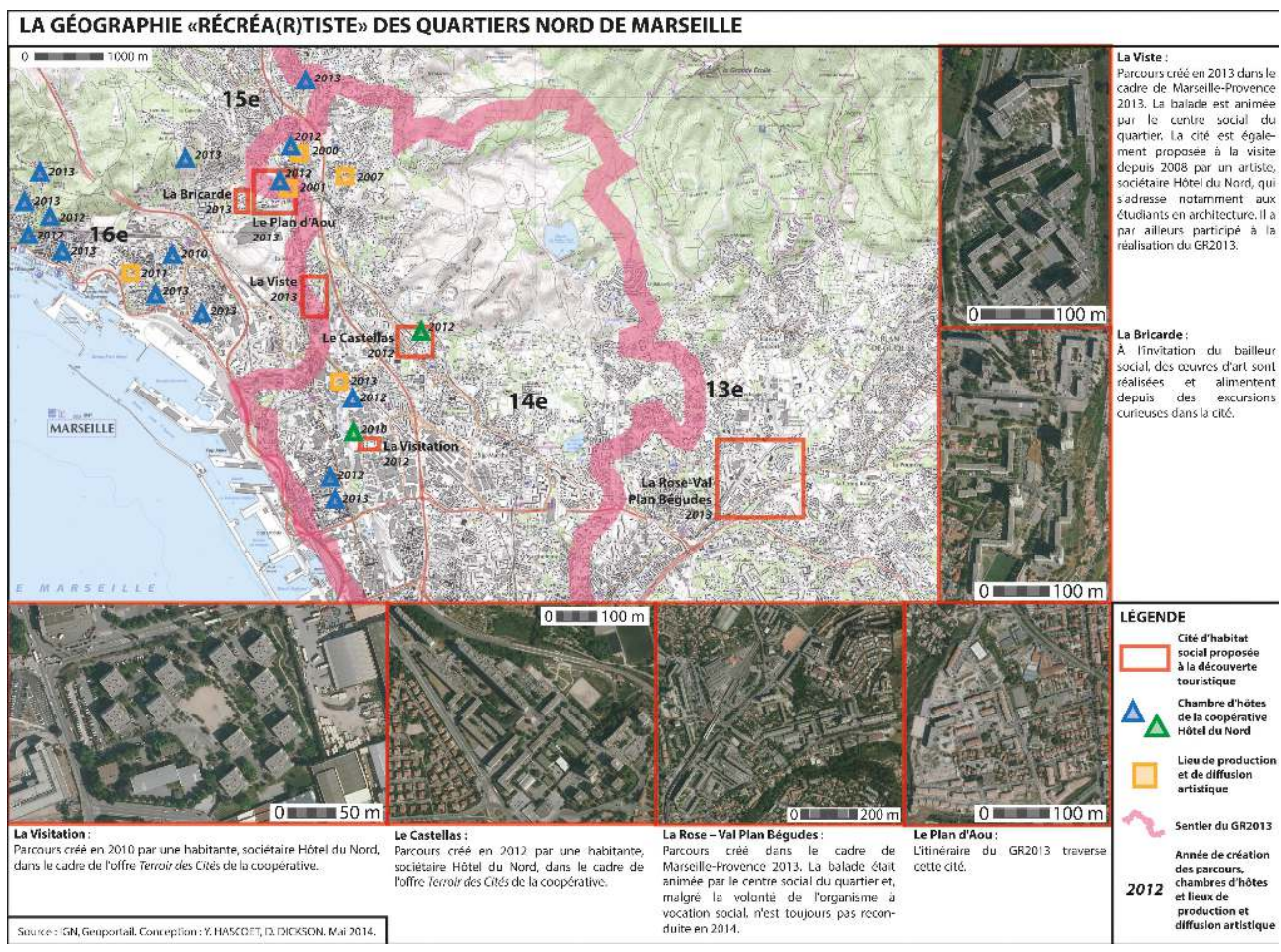

Cette carte localise les chambres d'hôtes Hôtel du Nord, six cités d'habitat social concernées par un mode découverte touristique et/ou un projet artistique, et précise enfin le tracé du GR13.

Source : ING, Geoportail. Conception : Yannick Hascoët et D. Dickson

\section{Amateur/professionnel}

L'expression même «jouer les guides » renvoie au statut ambivalent des personnes qui prennent en charge la visite. Ce ne sont évidemment pas des guides touristiques professionnels, mais des habitants qui en même temps inventent et reproduisent une pratique touristique incontournable, celle de la visite. En l'espèce, le procédé par lequel l'habitant devient opérateur de la mise en tourisme naissante rappelle une référence, sans doute trop rapide et insatisfaisante à ce stade de l'analyse, mais dont il sera utile de mesurer à terme la valeur heuristique. Le tourisme des quartiers nord, métamorphosant en guide l'habitant de banlieue, renvoie en effet ce qui s'est passé dans le dernier tiers du XVIII ${ }^{\mathrm{e}}$ siècle au bord de la mer, un espace alors réputé dangereux et en voie de domestication. Alain Corbin (1988) et Jean-Didier Urbain (1994), dans leur travaux respectifs explorant les origines du tourisme balnéaire, ont montré comment une élite économique et culturelle, pionnière du bord de mer, a en effet transformé en guides et maîtres-baigneurs des pêcheurs appartenant à un groupe réputé rude et inhospitalier. De ce point de vue, donc, la figure de l'habitant-guide relèverait moins d'une pratique innovante que de la reconduction/reproduction des pratiques séminales des premiers temps touristiques. Ici, comme en d'autres temps et espaces, « naturels » ou artificialisés, les pratiques touristiques se greffent dans les marges - spatiales et sociales - et opèrent une requalification conjointe de lieux et de groupes sociaux jusqu'alors déconsidérés. 
11 Autre signe du caractère naissant de ce tourisme: l'absence d'itinéraires fermement arrêtés (la carte 2 privilégie ainsi un vague zonage pour cerner les contours de la balade urbaine). Une caractéristique essentielle de l'offre est en effet le caractère changeant du tracé, au gré des rencontres durant la balade et des souhaits des participants. Bien que ces modes opératoires compliquent l'enquête, cette difficulté renseigne néanmoins sur la nature très expérimentale de ce tourisme balbutiant. Si les discours durant la visite expriment clairement des réflexions d'ordres politique et social (actualité économicosociale, mutations urbanistiques et histoire du logement social, mémoires ouvrières et migratoires) - tenant plus ou moins à distance le registre touristique accoutumé -, l'amateurisme est prégnant dans la mise en forme. La souplesse observée vis-à-vis des canons usités de la visite guidée - ne pas dépasser le temps prévu ou ne pas dévier de l'itinéraire annoncé - n'est toutefois pas interprétée comme un défaut de prestation, mais plutôt comme la marque d'une certaine authenticité de la pratique.

Les « guides » en récusent d'ailleurs bien souvent le titre et se trouvent fort démunis pour s'auto-qualifier : "Je sais pas comment qualifier ça. » (Fadela, la trentaine, animatrice de balades offertes par le centre social de La Viste, habitante du quartier, formée par Hôtel du Nord, rencontrée en entrevue en février 2014.) Habitants authentiques, patrimoine méconnu, territoire vierge (ou du moins réputé peu fréquentable et fréquenté)..., autant d'éléments qui renvoient à la figure polarisée et polarisante du pionnier, du défricheur en quête de terrae incognitae. Sans compter que l'habitant métamorphosé en " guide » n'est pas le seul détenteur de la parole: la balade relève d'une co-construction et à tout moment chaque participant questionne, raconte, propose... Les codes de la visite guidée formelle sont donc ici mis à mal et ce tourisme ne s'affirme pas seulement hors des sentiers battus du point de vue spatial, aux franges de la ville, mais également par les modalités mêmes de son effectuation.

\section{Visiteur/visité}

13 Encore aujourd'hui, et même si les effets de Marseille Provence 2013 (MP2013) restent à mesurer et parasitent la lecture des publics, il demeure que les offreurs sont également bien souvent les demandeurs: nombre de participants aux balades urbaines ne sont autres que des sociétaires ou des sympathisants de la coopérative Hôtel du Nord qui proposent eux-mêmes des balades urbaines ou des chambres d'hôtes, parfois les deux : les visiteurs du jour pouvant être les visités de demain. Autrement dit, beaucoup des participants sont eux-mêmes des habitants des quartiers nord (sinon des Marseillais). Dans les quartiers de Val Plan Bégudes et de La Viste, où des animateurs des centres sociaux ont étés formés par Hôtel du Nord pour animer des balades (à l'approche de MP2013), le public reçu est, là aussi, bien souvent local (illustrations 4-5). En effet, composé pour moitié d'habitants des quartiers (« pour éviter l'effet zoo » dit-on au Centre social de Val Plan Bégudes), le reste du groupe provient, selon le directeur du Centre social de Val Plan Bégudes (rencontré en entrevue en janvier 2014), du centre-ville, du Vieux-Port et de la partie sud de la ville. Il évoque ainsi des «Marseillais qui viennent découvrir les quartiers nord ».

Une participante marseillaise défend en effet : « Il y a des choses à découvrir tout près, c'est pas la peine d'aller au bout du monde ${ }^{7}$. » Le tourisme des quartiers nord est donc un tourisme de grande proximité qui attire « beaucoup de Marseillais qui ne connaissent pas leur ville » (Loïc, sociétaire Hôtel du Nord, retraité de l'Inspection des affaires sociales et 
sanitaires, militant au Parti communiste français, rencontré en entrevue en juin 2013). La faible distance spatiale entre les espaces quotidien et hors quotidien (cas des Marseillais du centre-ville qui explorent son nord), parfois l'abolition de la distinction (cas de l'habitant qui redécouvre son propre quartier à l'occasion d'une balade), rend dès lors le couple ici/ailleurs autrement mobilisable. Ce tourisme des quartiers élabore et réinjecte une altérité à quelques encablures de chez soi, voire au sortir du palier de son immeuble : "J'ai un œil différent. C'est vrai que des fois on traverse une rue, un endroit, on n'observe plus rien. Là, je regarde les pierres, je regarde les murs... je regarde différemment. Il est beau à visiter mon quartier. " (Christelle, la cinquantaine, habitante de la cité de La Visitation depuis 1988, sans-emploi, sociétaire Hôtel du Nord et guide de son quartier, rencontrée en entrevue en juin 2013). Si Jean-Didier Urbain, discutant l'exotisme, note que « l'invisibilité naît de l'usure du regard », « de cette perte de vigilance que produisent familiarité et répétition» (2003: 102), il apparaît bien que ce tourisme de grande proximité, offrant le cadre d'un changement d'optique selon une «exotisation du proche » (Matthey, 2007), constitue le moteur de la (re)découverte.

Illustration 4 : Balade urbaine capitale

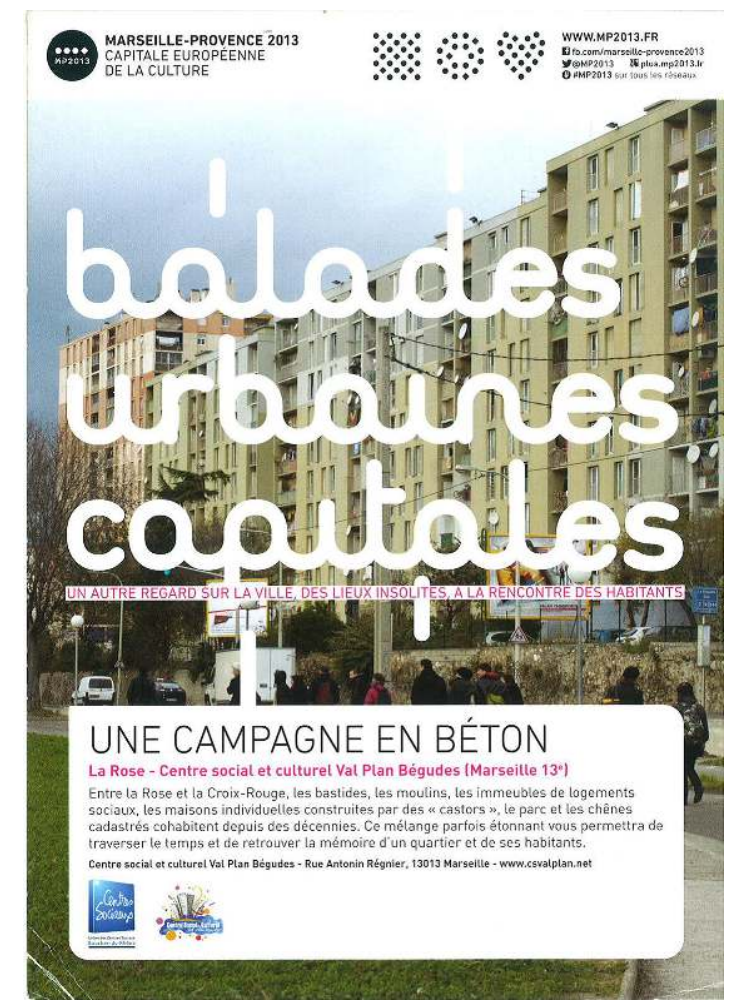

Dépliant faisant la promotion de la « balade urbaine capitale » proposée par le Centre social de Val Plan Bégudes dans le cadre de MP13. "Un autre regard sur la ville, des lieux insolites, à la rencontre des habitants », y vante-t-on. Un autre regard sur « sa » ville, des lieux insolites au sortir de «chez soi », à la rencontre « de ses voisins », tout compte fait.

Source : Marseille-Provence 2013 


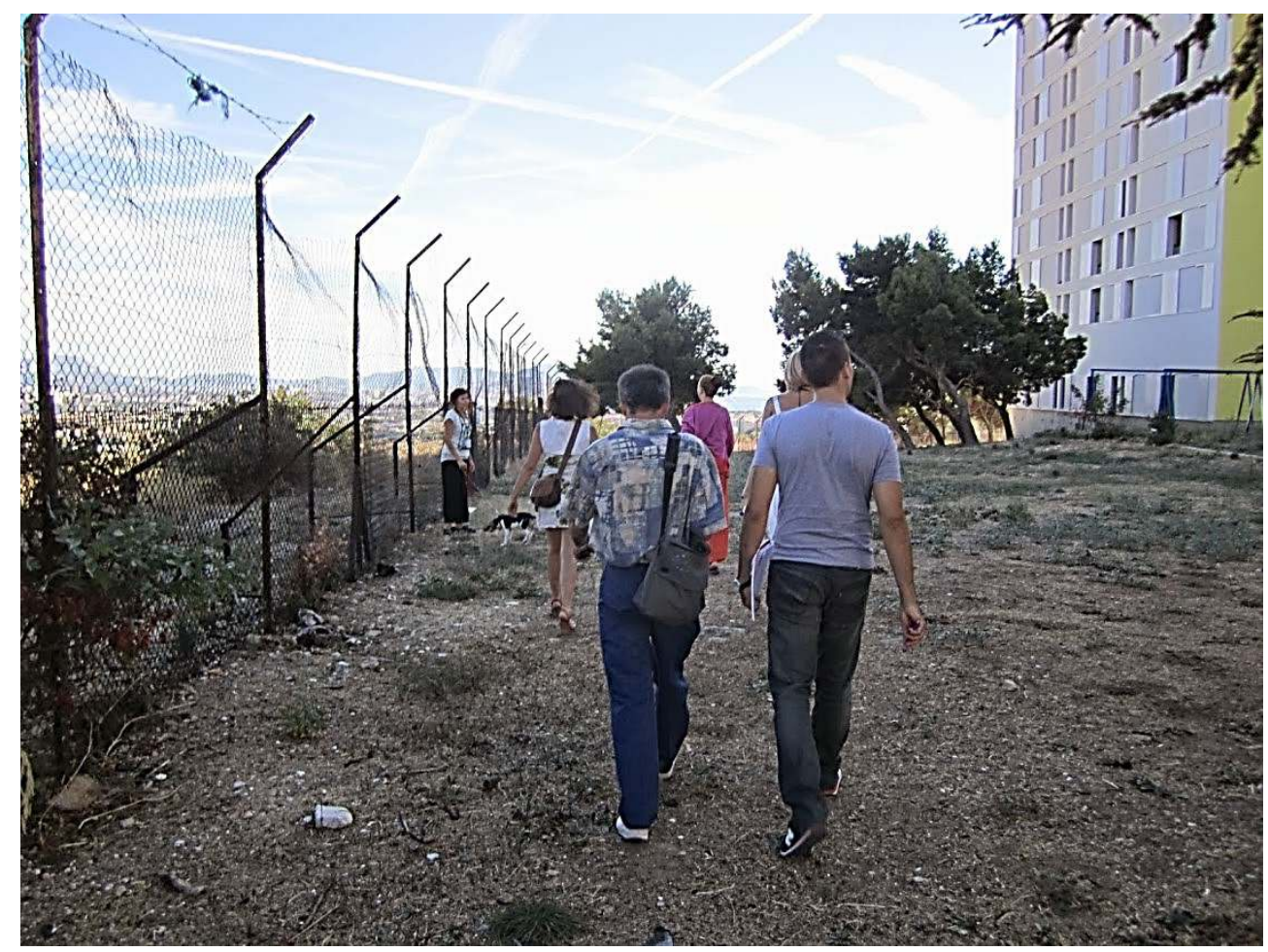

Un petit groupe au détour de la cité La Viste, en balade avec Sandrine (salariée du centre social) et Fadela (salariée du centre social et habitante du quartier). Initiée avec MP13, la balade est depuis proposée par le centre social du quartier

Photographie : Alice Mash, septembre 2013

\section{Centre/périphérie}

Actuellement, au sein d'Hôtel du Nord seulement deux chambres d'hôtes en cité d'habitat social existent (chez deux guides, Christelle et Julie). Cette offre pose problème dans la mesure où la réglementation interdit la location d'une chambre d'hôtes en logement social. Le collectif cherche aujourd'hui à faire évoluer la législation, révélant par là même combien ces offres bousculent le droit du tourisme ${ }^{8}$. En attendant, la plupart des chambres proposées le sont dans des maisons situées dans les anciens noyaux ouvriers ou dans des bastides provençales et bourgeoises, soit des lieux de villégiature somme toute classiques et, d'ailleurs, pour une bonne part, situés du côté de l'Estaque (carte 2), seul espace des quartiers nord touristiquement reconnu et porteur d'une imagerie "petit village de pêcheurs ». Emblématisé par de célèbres peintres impressionnistes, cubistes et fauvistes (Cézanne, Braque, Renoir, Dufy, etc.), le quartier de l'Estaque échappe à la marginalisation des quartiers nord - dont il fait néanmoins administrativement partie et bénéficie de toutes les attentions des entreprises touristiques publiques et privées. Du reste, si originalité il y a, c'est beaucoup moins au cœur des cités d'habitat social (deux chambres, deux balades dédiées) que dans les nuitées proposées en roulotte, dans une maison inscrite sur la liste des Monuments historiques ou sur un voilier (amarré dans le port de l'Estaque). Se conjuguent ainsi hébergement « insolite » et haut lieu touristique.

Ces observations invitent dès lors à nuancer l'idée que le tourisme des quartiers nord serait un tourisme invariablement «alternatif ». Plus encore, il ne faudrait pas l'analyser 
en termes d'alternative au tourisme. Il fonctionne davantage sur un modèle dialectique entre centre et périphérie, hauts lieux du tourisme et lieux décalés du regard touristique classique. Ce tourisme joue sur plusieurs tableaux, à cheval entre le in et le off. Après avoir visité les lieux phares - Notre-Dame de la Garde, le Musée des civilisations de l'Europe et de la Méditerranée (MUCEM), les calanques -, ce jeune couple de Picardie en visite à la cité de La Visitation explique : «On veut aussi voir les autres quartiers. Ce qui n'est pas la vitrine de Marseille. C'est aussi là que vit une partie de la population » (échange à l'occasion d'une balade animée par Christelle à la cité de La Visitation, juin 2013).

Au demeurant, les offreurs eux-mêmes revendiquent faiblement le caractère insolite de leur démarche: c'est l'étiquette "touristique " même qui est discutée, séparant les partisans d'une action assumée dans le champ de l'économie touristique d'autres, en mal de détermination par rapport à cette qualification touristique, même conçue autour de valeurs éthiques. Pour le dire autrement, plusieurs de ces offreurs sont dans l'incapacité de qualifier leur activité. Une partie des sociétaires mettent en doute l'intérêt de se rapprocher du secteur touristique, oscillant entre interrogation et récusation de l'identité touristique de leurs pratiques. Un jour, une sociétaire s'est élevée en faux par rapport au terme de "location en chambre d'hôtes »: elle prétendait "offrir l'hospitalité " au demeurant... payante (échange capté lors d'une journée organisée par Hôtel du Nord à destination d'éventuels partenaires du secteur touristique, sur les enjeux du tourisme responsable, juin 2014).

18 Cette indétermination, dont les paradoxes s'expriment au creux des propos d'une partie des membres d'Hôtel du Nord, caractérise un positionnement dans le champ de l'offre en tension entre valeurs et projets « autres » et modes opératoires effectifs. Entre un « pour soi » du projet et « en soi » de l'objet.

\section{De la diversité des champs d'intervention}

\section{Champ du tourisme, champ du militantisme}

19 Pour réaffirmer l'hypothèse d'un tourisme moins alternatif que complémentaire de l'offre établie, il convient de rappeler que les ouvertures de chambres se sont accélérées dans la perspective d'une manifestation porteuse d'opportunités et somme toute très officielle : MP2013. La coopérative Hôtel du Nord «flirte » avec les frontières du tourisme - urbain, solidaire, insolite - et il devient dès lors délicat d'en identifier et d'en qualifier les prestations. S'agit-il d'une offre réellement d'un nouveau genre, d'une nouvelle hybridation, ou faut-il y voir davantage les effets de l'extension, dans un quartier d'habitat social, de modes opératoires déjà connus?

Un bon nombre des membres fondateurs de l'organisation récusent une quelconque appartenance à l'économie touristique (en tout cas comme domaine principal de compétences), alors même que le vocabulaire lui soit emprunté (hospitalité, chambres d'hôtes, balades urbaines...). Une ambivalence très justement pointée par Camille Rouchi (2013), qui fait état des propos contradictoires de l'historienne à l'origine du projet qui déclare d'abord: «ça n'a rien à voir avec du tourisme » et qui affirme plus tard: « on ne peut pas fonctionner sans l'imbrication tourisme et patrimoine, elle est naturelle». D'autres, en revanche, préconisent plus nettement des rapprochements avec ce secteur, à condition qu'il soit estampillé "éthique». La coopérative est donc à un tournant: continuer de fonctionner à la marge, au risque d'un vase trop clos des publics et par 
conséquent des limites financières du projet, ou affirmer sa dimension touristique, au risque de la dilution des idéaux initiaux dans un système touristique qui se réinvente continûment (et de façon de plus en plus rapide depuis un quart de siècle). Quoique localement le Service du tourisme de la Ville ait d'autres préoccupations, plus rentables :

Ce que je cherche pour Marseille, c'est faire venir des devises. L'accent est mis dans deux voies qui sont directement très productives: le tourisme d'affaires et les croisières. Les quartiers nord, on ne s'y est pas du tout attelé, envoyer des touristes là-bas, déjà que dans certaines cités la police n'y met plus les pieds. Je sais qu'il y a eu des balades, des initiatives avec MP13 mais... c'est sûrement pas bien mais... non c'est pas du tout notre préoccupation mais après manifestement ça intéresse des gens alors... (Directeur du Service de tourisme de la ville, rencontré en entrevue en janvier 2014.)

Il demeure que la ligne de partage entre les pro-tourisme et les touristico-sceptiques demeure poreuse ; au sein même de l'espace démocratique d'Hôtel du Nord, la discussion va bon train. Chacun semble avoir conscience des possibilités offertes par l'affirmation touristique et une crainte, bien légitime :

on pourrait juste devenir au service d'un tourisme un peu différent... et à un moment donné on peut très bien devenir comme beaucoup de mutuelles sont devenues. C'est-à-dire... il y a des banques qui au départ étaient des mutuelles, qui sont devenues des banques comme les autres, mises à part qu'elles brandissent le petit drapeau «mutuelle » de temps en temps, mais c'est du bidon. (Christophe, la cinquantaine, philosophe-enseignant à l'École des beaux-arts de Marseille, sociétaire Hôtel du Nord, rencontré en entrevue en juin 2013.)

Nonobstant l'ambivalence d'une offre à la fois «traditionnelle» et «nouvelle», les attentes des publics désireux de sortir des sentiers battus sans perdre de vue les hauts lieux constituent un élément de développement effectif des pratiques touristiques des quartiers nord. Pour les acteurs de ces offres, la démarche doit permettre l'exploration d'altérités négligées ou refoulées et faire connaître/reconnaitre des individus et un territoire profondément maltraité dans les représentations sociales. Caractérisés par un volontarisme éthique fort, les membres de l'organisation se reconnaissent à la gauche de l'échiquier politique : «Bien sûr qu'Hôtel du Nord c'est des gens de gauche. Tout le monde s'en fout sinon des quartiers nord et de ses patrimoines. » (Marseillaise, enseignante de français en collège retraitée, habitante du sud de la ville, la soixantaine, rencontrée lors d'une balade urbaine à Le Canet en juin 2013, qui a découvert Hôtel du Nord dans le magazine culturel français Télérama.) La forme même du collectif, une SCOP, et son ambition affichée de permettre à terme un complément de revenus aux ménages les plus précarisés s'engageant dans la voie de l'hospitalité (par l'offre d'une chambre ou d'une balade) renseignent sur cette dimension politique du projet.

\section{Champ du tourisme, champ de l'art}

Encore indécis entre projet touristique ou projet politique - à moins qu'il ne soit les deux - le tourisme des confins de la métropole marseillaise flirte également avec le monde artistique. Deux initiatives, que l'on peut qualifier de pratiques « récréa(r)tistes ", mettent en effet sur la piste d'une autre hybridation, dont la « nouveauté » est ici encore fort ancienne. Pratiques artistiques et touristiques se sont en effet alimentées mutuellement de longue date, que l'on pense par exemple au rôle du mouvement romantique dans l'émergence du désir de rivage (Corbin, 1988). 
Formant une boucle de 365 kilomètres et explorant le périurbain (friches industrielles, lotissements pavillonnaire, décharges, grands ensembles, sites « naturels »...), le GR2013, projet phare de MP2013 que l'on propose de qualifier comme une dérive métropolitaine fléchée (Debord, 1956), fut porté par un éditeur marseillais (parution d'un guide) avec l'idée de «tracer le logo de la métropole avec nos pieds » (Bastien, la trentaine, éditeur, passionné d'écologie, habitant du quartier du Panier, qui a fait des classes préparatoires en lettres, rencontré en entrevue en janvier 2014). Les « artistes-marcheurs ${ }^{9}$ » chargés du tracé ont de fait matérialisé un usage inédit des lisières de la ville et l'on peut supposer que cette mise en scène (en schème pourrait-on dire) nourrisse une esthétique nouvelle et participe à la motivation de touristes s'aventurant sur leurs traces. Cette hypothèse s'adosse bien sûr au principe d'une artialisation (Roger, 1997) comme transmutation d'un "pays» en "paysage», que celle-ci s'effectue in situ (land art, jardins) ou in visu (photographie par exemple). Ce mécanisme de valorisation esthétique de (mi)lieux jusqu'ici ignorés (mais dotés au passage de valeur marchande) est amplifié par «le rôle croissant des médias du tourisme et des vacances » comme le remarquait déjà dans les années 1970 Yves Lacoste (1977 : 66). De même que « l'affreux pays » du bord de la mer ou de la montagne devint un " paysage sublime » (et partant encapsulé par l'acculturation et l'économie touristiques) sous l'effet d'un système de valeurs et de modèles artistiques promu par une élite artiste (Heinich, 2005), le paysage des confins métropolitains pourrait devenir culturellement et symboliquement - voire économiquement - monétisé. L'expérience conduite à la cité de la Bricarde, grand ensemble d'habitat social, en exemplifie les possibilités.

À l'initiative du bailleur social du quartier (Logirem) et en vue de MP2013, le quartier abrite depuis 2009, au fil des résidences d'artistes, des installations contemporaines (illustrations 6-7) donnant à la cité, jusque-là davantage associée au thème politicomédiatique de «la crise des banlieues ", des allures de "Cité des curiosités » (nom du projet), ce qui ne manque pas d'alimenter le développement de mobilités curieuses, bien qu'elles échappent aux radars de l'économie touristique classique. À des échelles différentes (de la métropole au quartier d'habitat social) mais toujours relativement décalés dans le lieu (les franges urbaines) et l'intention (faire art in situ), ces exemples de pratiques que nous appelons "récréa(r)tistes" invitent à re-poser la filiation entre créations artistiques et pratiques touristiques. Dans le domaine littéraire, depuis François Maspero avec Les passagers du Roissy Express paru en 1990, l'accroissement d'une littérature sur les lisières urbaines compose également un corpus intéressant pour suivre une telle piste ${ }^{10}$. 
Illustrations 6 : « L'Envol » (2011) de Jean-Marc Munerelle

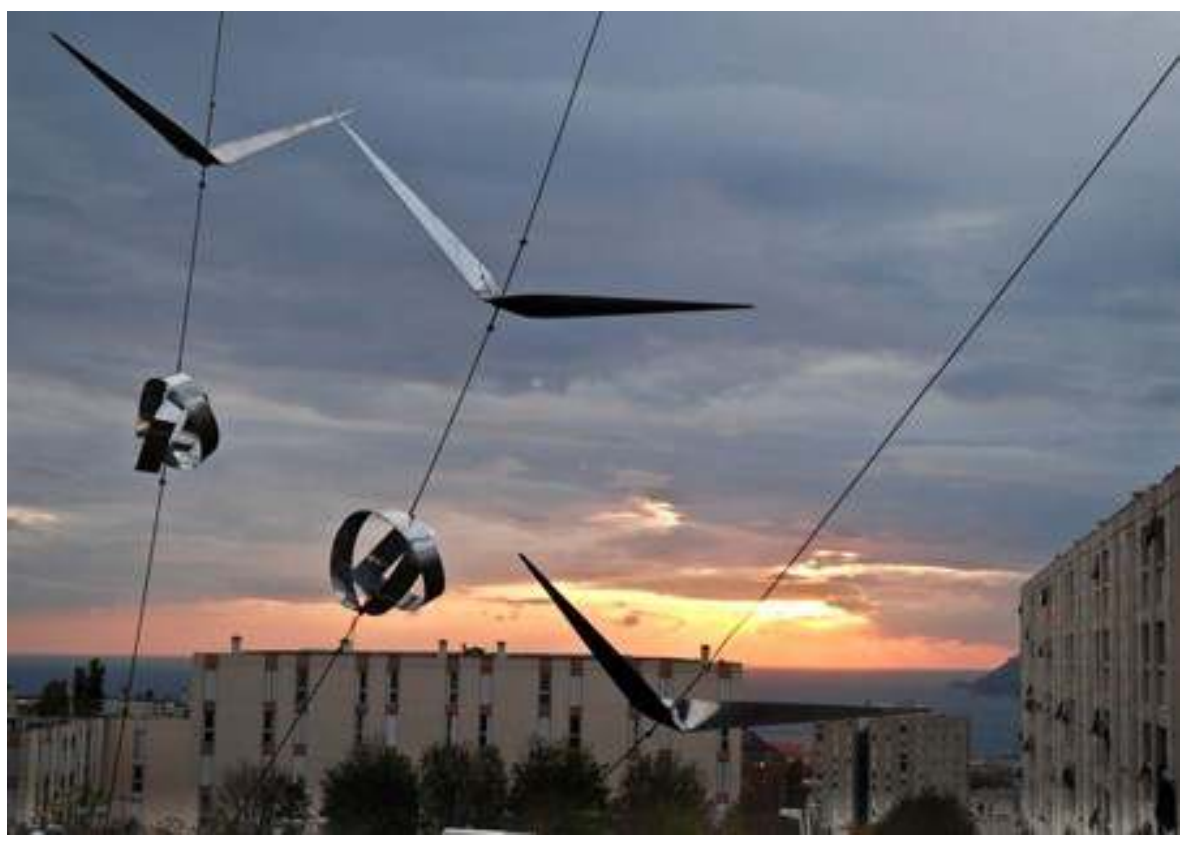

Une œuvre d'art contemporain au cœur d'une cité des quartiers nord de Marseille.

Photographie : Munerelle

Illustration 7 : «Fin » (2012) par le duo d'artistes Gethan \& Myles

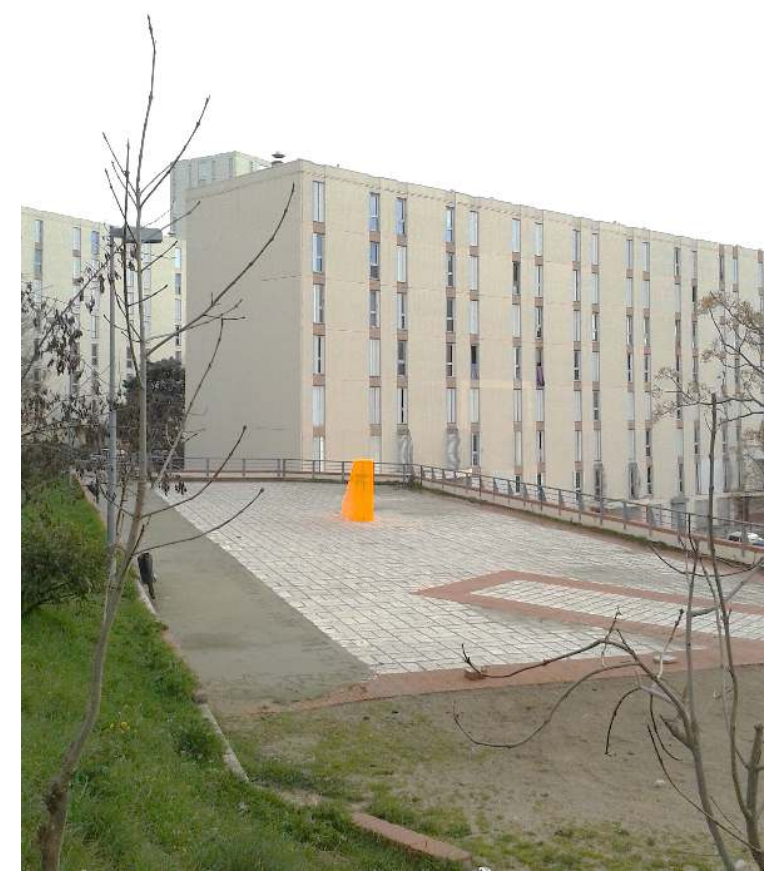

Une deuxième œuvre d'art contemporain au cœur d'une cité des quartiers nord de Marseille. Photographie : Yannick Hascoët, février 2014 


\section{Conclusion} œuvre et parce que les catégorisations usuelles ont montré leurs limites opératoires, participe d'une ré-interrogation en profondeur des ressorts et des modalités du fait touristique. Sans prétendre trancher ici sur des questions fondamentales renvoyant aux théories de longue portée sur la nature des société contemporaines (postmodernité, Lyotard, 1979; hypermodernité, Lipovetsky, 1983), les travaux de Jean Viard (2000) peuvent apporter un premier éclairage heuristique. Pour ce dernier, le « post-tourisme » caractérise des processus où attractivité touristique d'un lieu et itinéraires résidentiels sont conjointement articulés: de nouveaux résidents, qualifiés de "post-touristes", parce qu'ils ont déjà fréquenté ces lieux ou d'autres de même type comme touristes, sont attirés par des environnements qui ne sauraient plus être (ou plus seulement en tous cas) reconnus comme typiquement touristiques. En Angleterre, John Urry (1990) est d'ailleurs allé plus loin dans l'analyse des convergences/mixités entre pratiques. Alors que le tourisme moderne s'est structuré à partir d'une différenciation nette des temps sociaux (temps de travail par rapport à temps de récréation), l'observation d'une «dédifférenciation accoucheuse » d'une mise en tourisme généralisée, en tous temps et lieux, conduit Urry à caractériser les manifestations contemporaines de postmodernité touristique. Toutefois, pour globale qu'elle soit, cette dernière perspective amalgame sortie du tourisme et après-tourisme de masse (Girard, 2013). Or, les pratiques qu'illustrent les quartiers nord de Marseille ne remplacent d'aucune manière les pratiques de tourisme de masse. Elles les complètent - spatialement, culturellement -, participent d'un recyclage toujours plus extensif des marges (géographiques et sociales) pour dessiner les lignes les plus avancées des tendances contemporaines (Lipovetsky et Serroy, 2013). Ce faisant, les terrains ici explorés rendraient compte davantage d'un « hyper-tourisme ", exemplifiant les extensions et les recyclages, culturels et marchands, de pratiques touristiques inscrites dans le temps long de leur histoire moderne. En l'état, parcours touristiques et itinéraires artistiques réintègrent, effectivement et symboliquement, les quartiers nord de Marseille dans l'offre culturelle locale et le territoire métropolitain.

Ce constat intermédiaire n'est d'ailleurs pas sans soulever des problèmes connexes mais fondamentaux et congruents à la qualification de tourisme hypermoderne. Le premier est celui de la nouvelle valeur marchande de ces territoires: les promoteurs immobiliers intervenant sur les ruines de la rénovation urbaine au nord de Marseille ne s'y trompent pas et l'attractivité émergente des quartiers nord n'est pas sans risque pour les populations les plus socio-économiquement fragiles. Le second est celui de l'indigénisation. Même éthiquement motivé et " participatif », ce tourisme laisse, en fait, une grande majorité d'habitants en marge des initiatives, bien plus « objets » de la visite qu'acteurs de leurs cités. Quand les discours politico-médiatiques véhiculent à l'envi le registre des " cités interdites », des « îlots d'insécurité » et des «territoires perdus » (de la République), les initiatives et les nouvelles offres des quartiers nord de Marseille, pour politiquement solidaires qu'elles soient, peuvent-elles constituer plus qu'une démarche de cosmisation (mise en ordre et mise en esthétique) du « désordre » des cités? 


\section{BIBLIOGRAPHIE}

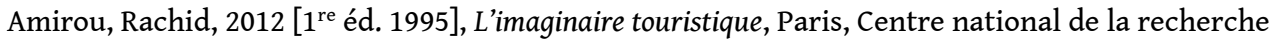
scientifique (CNRS).

Avenel, Cyprien, 2004, Sociologie des « quartiers sensibles », Paris, Colin.

Bourdieu, Pierre, 1979, La distinction. Critique sociale du jugement, Paris, Minuit.

Chapuis, Amandine, 2009, «Quand le tourisme solidaire s'invite dans les métropoles du Nord.

L'exemple de la mise en tourisme du quartier parisien de Belleville », dans Jean-Pierre Lemasson et Violier Philippe (dir.), Destinations et territoires (vol. 2), « Tourisme sans limites », Québec, Presses de l'Université du Québec

Chomette, Guy-Pierre, 2014, Le piéton du Grand Paris, Paris, Parisgramme.

Corbin, Alain, 2010 [ $1^{\text {re }}$ éd. 1988], « Le territoire du vide. L'Occident et le désir de rivage », Paris, Flammarion.

Debord, Guy, 1956, "Théorie de la dérive », Les lèvres nues, n 9 (accessible sur : La Revue des Ressources, <http://www.larevuedesressources.org/theorie-de-la-derive,038.html>, consulté le 7 février 2016).

Girard, Alain, 2013, « Faut-il raccorder une théorie générale de la post-modernité à une théorie à moyenne portée du post-tourisme ? ", dans Hugues François, Philipe Bourdeau et Liliane PerrinBensahel (dir.), Fin(?) et confins du tourisme, Interroger le statut et les pratiques de la récréation contemporaine, Paris, L'Harmattan.

Heinich, Nathalie, 2005, L'élite artiste. Excellence et singularité en régime démocratique, Paris, Gallimard.

Jolé, Michèle, 2012, « Hôtel du Nord. La construction d'un patrimoine commun dans les quartiers nord de Marseille », Métropolitiques, 4 janvier, <https://www.metropolitiques.eu/Hotel-du-NordLa-construction-d-un.html>, consulté le 7 février 2016.

Lacoste, Yves, 1977, « À quoi sert le paysage ? Qu'est-ce qu'un beau paysage ? ", dans Alain Roger (dir.), 1995, La théorie du paysage en France (1974-1994), Seyssel, Champ Vallon.

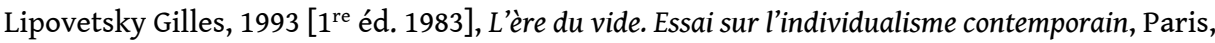
Gallimard.

Lipovetsky, Gilles et Jean Serroy, 2013, L'esthétisation du monde. Vivre à l'âge du capitalisme artiste, Paris, Gallimard.

Lyotard, François, 1979, La condition postmoderne, Paris, Minuit.

Maspero, François [photographies Frantz Anaïck], 2004 [1 ${ }^{\text {re }}$ éd. 1990], Les passagers du Roissyexpress, Paris, Seuil.

Matthey, Laurent, 2007, «Si proche, si loin! Penser les processus urbains à partir des modèles de la géographie du tourisme? ", Articulo, n 3, <http://articulo.revues.org/613>, consulté le 7 février 2016 
Pouvreau, Benoît, 2011, « Faut-il 'patrimonialiser' les grands ensembles? », Métropolitiques, 28 mars, <http://www.metropolitiques.eu/Faut-il-patrimonialiser-les-grands.html>, consulté le 7 février 2016.

Roger, Alain, 1997, Court traité du paysage, Paris, Gallimard.

Roncayolo, Marcel, 2014 [1 ${ }^{\text {re }}$ éd. 1990], L'imaginaire de Marseille. Port, ville, pôle, Lyon, École normale supérieure (ENS).

Rouchi, Camille, 2013, Révéler le patrimoine des quartiers nord de Marseille : entre expérimentation européenne et promotion du territoire, mémoire de master professionnel en tourisme, Université Paris 1 - Panthéon-Sorbonne.

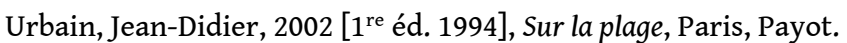

Urbain, Jean-Didier, 2003, Ethnologue mais pas trop, Paris Payot.

Urry, John, 2002 [1 $1^{\text {re }}$ éd. 1990], The Tourist Gaze, Londres, Sage.

Vayssière, Bruno, 2002, " Pour une patrimonialisation délibérée des grands ensembles ", entretien, dossier « Le grand ensemble, histoire et devenir », Urbanisme, $n^{\circ} 322$, janvier-février.

Veschambre, Vincent, 2013, « Un nouveau regard sur les grands ensembles ? », Urbanisme, dossier «Que faire des années $1970 ? », n^{\circ} 388$.

Viard, Jean, 2000, Court traité sur les vacances, les voyages et l'hospitalité des lieux, Paris, L'Aube.

\section{NOTES}

1. <http://h2h.hoteldunord.coop/fr/hotel-du-nord/>, consulté le 7 février 2016.

2. <http://hoteldunord.coop/balades/la-balade-de-christiane-a-la-visitation/>, consulté le 7 février 2016.

3. Initiées en France en 1984, les Journées européennes du patrimoine réunissent à présent une cinquantaine de pays, qui organisent une fois l'an des manifestations culturelles et ouvrent bien souvent des lieux exceptionnellement proposés à la visite.

4. Le GPU, remplacé en 2007 par le Contrat urbain de cohésion sociale (CUCS), est un outil de mise en œuvre de la politique de la Ville en faveur de quartiers socio-économiquement fragiles et, à ce titre, identifiés comme nécessitant une action ciblée de l'action publique.

5. Marseille Provence 2013 (MP2013) désigne une vaste série d'événements culturels majeurs ayant eu lieu à Marseille et dans le département des Bouches-du-Rhône au moment de l'élection de Marseille au titre de « capitale européenne de la culture».

6. Un tiers est constitué de personnes morales (entreprises et associations) et une poignée de sociétaires soutiennent les actions sans être nécessairement habitants ou acteurs de terrain. En sus des chambres et des balades, la vente de productions locales (récits, savon de Marseille, miel, etc.) complète le dispositif. Enfin, historiquement centrée sur les quartiers nord de la ville $\left(13^{\mathrm{e}}\right.$, $14^{\mathrm{e}}, 15^{\mathrm{e}}$ et $16^{\mathrm{e}}$ ), la SCOP travaille également sur les $2^{\mathrm{e}}$ et $3^{\mathrm{e}}$ arrondissements (voire à l'échelle métropolitaine, à Vitrolles), d'autres mairies ayant depuis emboîté le pas au $8^{\mathrm{e}}$ secteur de Marseille dans la signature de la Convention de Faro.

7. Extrait du clip promotionnel «Balades urbaines capitales, six parcours thématiques à Marseille et Miramas » : <https://www.youtube.com/watch?v=8qPf-IBL2iA>, consulté le 7 février 2016.

8. Hôtel du Nord, avec Accueil banlieue et le CDT 93, demandent à pouvoir expérimenter l'hospitalité en habitat social. Le collectif a saisi parlementaires et bailleurs sociaux en ces termes: "L'expérimentation législative locale est l'autorisation donnée par une loi à une collectivité territoriale d'appliquer une politique publique ne faisant pas partie de ses 
attributions légales, pour une période donnée. "L'expérimentation législative a comme but d'étudier les effets d'une réforme ou d'une loi sur un échantillon de personnes et dans un temps limité. La proposition est d'expérimenter la modification du deuxième alinéa du II de l'article L442-8-1 du code de la construction et de l'habitation, en insérant l'alinéa suivant : « une partie de leur logement comme chambre d'hôte sous réserve de l'accord préalable du bailleur social et dans la limite d'un nombre annuel de nuitées fixé préalablement » (extrait d'une note transmise par Hôtel du Nord à des parlementaires). (Voir <https://www.legifrance.gouv.fr/ affichCodeArticle.do?cidTexte=LEGITEXT000006074096\&idArticle=LEGIARTI000020459230>, consulté le 7 février 2016.)

9. Également auto-qualifié de « cercle des marcheurs » : c'est « le nom que se sont donné en 2012 une dizaine d'individus lorsqu'ils ont réalisé qu'ils formaient une scène et qu'ensemble ils travaillaient mieux que séparément. Installés à Marseille, Nantes ou Paris, les fondateurs du cercle sont des artistes, des marcheurs et des artistes-marcheurs. Cette scène, qui s'est formée à Marseille dans les années 2000, a directement inspiré l'invention du sentier métropolitain GR2013 pour la capitale européenne de la culture. » Ils partagent entre autres l'idée que l'art se passe d'abord dehors, et que des espaces apparemment aussi ordinaires que le monde périurbain valent tous les musées institués. <http://lecercledesmarcheurs.org/qui>, consulté le 7 février 2016.

10. Citons par exemple Le Piéton du Grand Paris de Chomette, paru en 2014, dans lequel l'auteur fait le récit d'un voyage de 300 kilomètres sur les traces du futur Grand Paris Express.

\section{RÉSUMÉS}

Le tourisme a toujours fonctionné par extension : les lisières d'hier sont aujourd'hui des hauts lieux touristiques, que l'on pense par exemple au bord de la mer, longtemps déprécié. Sur le terrain contemporain de la ville, ce sont les quartiers populaires péricentraux qui sont à présent saisis par un engouement et une économie touristiques ; plus inédite encore, la découverte par les touristes des quartiers populaires nettement périphériques. C'est le cas à Marseille, dont les quartiers nord, espaces emblématiques de la "crise des banlieues ", sont désormais l'objet d'un «front» touristique et patrimonial, animé par une coopérative d'habitants particulièrement active. Cette problématique de l'émergence d'un nouveau lieu d'intérêt touristique se fait particulièrement saillante en ce qui concerne des quartiers d'habitat social en hauteur, nombreux dans ces quartiers nord. Les grands ensembles, en effet, symboles de l'urbanisme moderne honnis des décennies 1950, 1960 et 1970, mobilisent eux aussi de nouvelles pratiques. Tantôt touristiques, tantôt artistiques, elles se combinent parfois : l'article présente ces pratiques « récréa(r)tistes » d'un nouveau genre. Plus largement, il s'agit de rendre compte et d'analyser le statut, la fonction et les enjeux de pratiques réputées à l'ombre du tourisme urbain institué.

\section{INDEX}

Mots-clés : marges touristiques, « front » touristique et patrimonial, quartiers nord de Marseille, grands ensembles populaires, pratiques « récréa(r)tiste». 


\section{AUTEURS}

\section{YANNICK HASCOËT}

Doctorant, EVS-RIVES (ENTPE), Université de Lyon ; yannick.hascoet@entpe.fr

\section{ISABELLE LEFORT}

Professeure, EVS-IRG (Lyon 2), Université de Lyon ;

isabelle.lefort@univ-lyon2.fr 